\title{
Anesthetic Management of Moyamoya Syndrome Secondary to Sickle Cell Anemia
}

\author{
Parmod K. Bithal1,๑ Ravees Jan ${ }^{2, \odot} \quad$ Ved P. Pandey ${ }^{2} \quad$ Parvaiz Ahmad ${ }^{2}$ \\ ${ }^{1}$ Division of Neuroanesthesia, Department of Anesthesia and \\ Perioperative Medicine, King Fahad Medical City, Riyadh, Kingdom \\ of Saudi Arabia \\ 2Department of Anesthesia and Perioperative Medicine, King Fahad \\ Medical City, Riyadh, Saudi Arabia

\begin{abstract}
Address for correspondence Ravees Jan, MBBS, MD, EDAIC, CHRM, Department of Anesthesia and Perioperative Medicine, King Fahad Medical City, Riyadh 11525, Saudi Arabia (e-mail: janravees@gmail.com).
\end{abstract}

J Neuroanaesthesiol Crit Care 2022;9:50-52.

\begin{abstract}
Keywords

- moyamoya syndrome

- sickle cell anemia

- normovolemia

- normocapnia

- normothermia

Moyamoya disease (MMD) is caused by stenosis or occlusion of internal carotid artery in brain, thereby reducing its blood supply. To augment blood flow, brain develops abnormal anastomotic vessels with deranged carbon dioxide reactivity and tendency to bleed. Moyamoya syndrome (MMS) is the name given to MMD when the latter results from secondary to some associated disease. Occurrence of MMS secondary to sickle cell anemia (SCA) presents unique challenges to neuroanesthesiologists. Management of various physiological parameters for cerebral revascularization surgery for MMD under general anesthesia necessitates vigilant and balanced control of various physiological variables, as the manipulation of a particular physiological variable for one pathology may adversely impact the same physiological variable for the associated disease, which will result in poor outcome of the patient. Therefore, optimum outcome of MMS is determined by a watchful balancing of various physiological parameters under anesthesia.
\end{abstract}

\section{Introduction}

Moyamoya disease (MMD) is a rare cerebrovascular disorder of narrowing of the internal carotid artery (ICA), which reduces cerebral blood flow $(\mathrm{CBF})$ and leads to the development of abnormal collateral vessels. Adults present with motor deficit, transient ischemic attacks, infarct, and intracranial hemorrhage. Children have ischemic events triggered by dehydration, fever, and even crying.

It may be primary (idiopathic) or secondary, also called Moyamoya syndrome (MMS), which results from an underlying pathology. ${ }^{1}$ When MMS secondary to sickle cell anemia (SS) presents for cerebral revascularization (CR), general anesthesia (GA) administration presents unique implications. We report anesthetic management of a MMS from SS scheduled for CR.

published online

November 14, 2021
DOI https://doi.org/

$10.1055 / \mathrm{s}-0041-1739349$

ISSN 2348-0548

\section{Case Report}

An 18-year-old, 60-kg male, SS patient presented with a history of seizures. Magnetic resonance angiogram (MRA) revealed narrowing of cervical segment of left ICA, with occlusion of supraclinoid segment and collateral development (Suzuki grade 3). History revealed that he received simple blood transfusions on multiple occasions in the past (including once for sickling crisis) but never exchange transfusion. He underwent uneventful cholecystectomy in the past. His medications consisted of hydroxyurea before admission and phenoxymethylpenicillin after admission. Cardiac and pulmonary systems were normal. His admission hemoglobin was $8.2 \mathrm{~g} / \mathrm{dL}$, with $60 \%$ sickle cell hemoglobin (HbSS). Other investigations were normal. Considering the issues associated with direct revascularization, the (c) 2021. Indian Society of Neuroanaesthesiology and Critical Care. All rights reserved.

This is an open access article published by Thieme under the terms of the Creative Commons Attribution-NonDerivative-NonCommercial-License, permitting copying and reproduction so long as the original work is given appropriate credit. Contents may not be used for commercial purposes, or adapted, remixed, transformed or built upon. (https://creativecommons.org/licenses/by-nc-nd/4.0/).

Thieme Medical and Scientific Publishers Pvt. Ltd. A-12, 2nd Floor, Sector 2, Noida-201301 UP, India 
neurosurgeon planned for encephaloduroarteriosynangiosis. His preoperative fasting period was covered with crystalloids infusion. One unit of packed red blood cells (PRBCs) was transfused the night before surgery, and another was transfused immediately after induction, raising hemoglobin to $10.3 \mathrm{~g} / \mathrm{dL}$. Following anesthesia induction with fentanyl and propofol and tracheal intubation with rocuronium, anesthesia was maintained with 50\% oxygen, sevoflurane, fentanyl, and rocuronium. Besides routine monitoring, radial artery was cannulated for monitoring blood pressure (BP) and blood gas analysis (BGA). Ventilation was targeted to arterial $\mathrm{CO}_{2}$ tension $\left(\mathrm{PaCO}_{2}\right)$ between 38 and $40 \mathrm{~mm} \mathrm{Hg}$. Normothermia was achieved with forced air warmer (FAW) and warmed fluids infusion. Normal saline and Ringer lactate were infused at $6 \mathrm{~mL} / \mathrm{kg} / \mathrm{h}$, and urine output and blood loss were replaced with crystalloids to maintain systolic BP between 130 to $140 \mathrm{~mm} \mathrm{Hg}$. Toward the end of procedure, paracetamol and granisetron were administered. The surgical procedure of 3.5 hours was smooth. Following reversal of rocuronium with sugammadex, trachea was extubated, and the awake patient was transferred to the neurointensive care unit, where to avert sickling, pain was managed with fentanyl, and satisfactory hydration was maintained. His postoperative period was uncomplicated.

\section{Discussion}

RBCs abnormalities in SS produce end-organ damage from microvascular occlusion. Surgery in sickle cell anemia (SCA) may produce vasoocclusive crisis (VOC), acute chest syndrome, cardiac failure, etc. ${ }^{2}$ When dealing with MMS from SS, the neuroanesthesiologist is faced with the challenges of delicately balancing various physiological parameters of two coexisting pathologies to circumvent harm to the patient. Principal perioperative concerns are to maintain $\mathrm{CBF}$ and steer clear of the triggers of sickling. Precautions to avoid sickling provocateurs must be adopted throughout the perioperative period.

An approach to avert complications of SCA is to reduce HbSS by preoperative transfusion. However, preoperative transfusion, either simple or exchange, remains contentious. ${ }^{3}$ Recently, though, preoperative transfusion has been recommended to increase hemoglobin to $10 \mathrm{gm} / \mathrm{dL}$ to keep HbSS below $30 \%$, which provides net benefit of good tissue oxygenation over downside of raised viscosity. ${ }^{4}$ Therefore, we transfused PRBCs preoperatively and also at induction, increasing hemoglobin to $10.3 \mathrm{~g} / \mathrm{dL}$.

Under anesthesia, focus should be on factors responsible for unfavorable outcomes in these patients. These factors, which either reduce CBF or trigger sickling, are as follows: hypotension, hypovolemia and dehydration (both increasing blood viscosity), hypoxia, $\mathrm{PaCO}_{2}$ alterations, and hypothermia.

BP management requires a tightrope walk since both low and high BP is detrimental. Low BP not only aggravates preexisting diminished $\mathrm{CBF}$ but, by reducing oxygen delivery, also encourages peripheral hypoxia and thereby sickling. Therefore, higher BP is desired in Moyamoya. Novel monitoring of EEG and near infrared spectroscopy monitoring during $C R$, to warn a decrease in $\mathrm{CBF}$ or to maintain optimum BP, are still in the investigative stage., ${ }^{5,6}$ Since BP in SS patients is generally lower than normal individuals, therefore higher BP enhances the stroke risk. ${ }^{7}$ Thus, avoid BP greater than 140/90 while maintaining good hydration to prevent $\mathrm{CBF}$ reduction. Hence, significance of invasive BP monitoring cannot be overstated. Vasopressors to augment BP are safe in MMD, but in MMS from SS, fluid loading is preferred over vasopressors because the latter promotes blood stasis and thereby sickling in periphery. ${ }^{8}$ Nevertheless, it is advocated to avoid precipitating a sickling event, treat hypotension expeditiously with fluids and vasopressor, as appropriate. ${ }^{9}$ Various studies have stressed on adequate hydration as the single compelling intervention to prevent hypotension for better outcomes of such patients.

Besides, poor hydration by increasing blood viscosity encourages erythrocytes polymerization in capillaries in SS. To prevent dehydration, we covered the perioperative period (including fasting period) with crystalloids infusion. Furthermore, we replaced urine output and blood loss with crystalloids. Since consensus is lacking on type of fluids, we infused both normal saline and Ringer's lactate. Postoperative vomiting is another potential source of dehydration, and we prevented that by prophylactic granisetron administration.

No study has demonstrated a direct link between perioperative hypothermia and sickling. ${ }^{2}$ In general, though, it is not unreasonable to consider hypothermia as a risk for VOC. Mild hypothermia for brain protection has been advocated during CR procedure, but it remains controversial. Therefore, normothermia should be standard of anesthetic care in SS patients. We achieved normothermia with FAW and warm fluids' infusion.

In MMS from SS, maintenance of normocapnia should be the goal because hypercapnia, as well as hypocapnia, decreases the $\mathrm{CBF}$ in regions perfused by collateral vessels, presumably because of intracerebral steal. ${ }^{10} \mathrm{PaCO}_{2}$ levels should be confirmed by BGA, and one should not rely on end-tidal $\mathrm{CO}_{2}$. Importantly, it is desirable to maintain good oxygenation because any episode of respiratory acidosis would make any accidental hypoxic episode more dangerous.

By adhering to the principles of preventing reduction of CBF and simultaneously instituting measures to avoid triggers of sickling, our patient had a smooth perioperative course.

In conclusion, successful anesthetic management of MMS secondary to SS was achieved by good hydration, stable hemodynamics, normocarbia and normothermia.

\section{Conflict of Interest}

None declared.

\section{Acknowledgment}

We are thankful to Research Centre at King Fahad Medical City. 


\section{References}

1 Scott RM, Smith ER. Moyamoya disease and moyamoya syndrome. N Engl J Med 2009;360(12):1226-1237

2 Firth PG, Head CA. Sickle cell disease and anesthesia. Anesthesiology 2004;101(3):766-785

3 Alotaibi GS, Alsaleh K, Bolster L, McMurtry MS, Wu C. Preoperative transfusion in patients with sickle cell disease to prevent perioperative complications: a systematic review and meta-analysis. Hematology 2014;19(8):463-471

4 Yawn BP, Buchanan GR, Afenyi-Annan AN, et al. . Management of sickle cell disease: summary of the 2014 evidence-based report by expert panel members. JAMA 2014;312(10):1033-1048

5 Vendrame M, Kaleyias J, Loddenkemper $\mathrm{T}$, et al. Electroencephalogram monitoring during intracranial surgery for moyamoya disease. Pediatr Neurol 2011;44(6):427-432
6 Lee JK, Williams M, Reyes M, Ahn ES. Cerebrovascular blood pressure autoregulation monitoring and postoperative transient ischemic attack in pediatric moyamoya vasculopathy. Paediatr Anaesth 2018;28(2):94-102

7 Pegelow $\mathrm{CH}$, Colangelo L, Steinberg M, et al. Natural history of blood pressure in sickle cell disease: risks for stroke and death associated with relative hypertension in sickle cell anemia. Am J Med 1997;102(2):171-177

8 Esseltine DW, Baxter MR, Bevan JC. Sickle cell states and the anaesthetist. Can J Anaesth 1988;35(4):385-403

9 Khurmi N, Gorlin A, Misra L. Perioperative considerations for patients with sickle cell disease: a narrative review. Can J Anaesth 2017;64(8):860-869

10 Oshima H, Katayama Y, Hirayama T. Intracerebral steal phenomenon associated with global hyperemia in moyamoya disease during revascularization surgery. J Neurosurg 2000;92(6):949-954 